\title{
ON THE DIOPHANTINE EQUATION $x^{m}=y^{n_{1}}+y^{n_{2}}+\cdots+y^{n_{k}}$
}

\author{
TOMOHIRO YAMADA \\ Department of Mathematics, Faculty of Science, Kyoto University, Kyoto, 606-8502, Japan \\ E-mail: tyamada@math.kyoto-u.ac.jp
}

(Received 31 March 2008; accepted 3 September 2008)

\begin{abstract}
We find all solutions of $x^{m}=y^{n_{1}} \pm y^{n_{2}} \pm y^{n_{3}} \pm y^{n_{4}}$ in integers $m, n_{1}, n_{2}, n_{3}, n_{4}$ for all relatively prime integers $x, y$ below 100 .

2000 Mathematics Subject Classification. 11A05, 11A25.
\end{abstract}

1. Introduction. In 1970, Senge and Strauss [5] proved that the sums of all digits of $n$ in bases $a$ and $b$ cannot be both small if and only if $\log a / \log b$ is irrational. Denote the sum of all digits in the canonical expansion of $n$ in base $a$ by $N_{a}(n)$. They proved that there are only finitely many integers $n$ with both of $N_{a}(n), N_{b}(n)$ below a fixed bound. Their proof is not effective, depending upon Mahler's generalization of Thue-Siegel-Roth theorem.

In 1980, Stewart [6] proved this result in an effective way, using Baker theory of linear forms in logarithms. Thus, in principle, we can effectively determine all integers $n$ satisfying $N_{a}(n), N_{b}(n) \leq k$ for any given $a, b, k$ such that $\log a / \log b$ is irrational.

Tijdeman and Wang [7], Wang [8] and Deze and Tijdeman [2] determined all integers $n$ with $N_{p}(n)+N_{q}(n) \leq 4$ for primes $p, q<200$. Indeed, they determined all solutions of the equation $x \pm y \pm z \pm w=0$ in integers $x, y, z, w \in S(p, q)$ for two primes $p, q<200$, where $S(p, q)$ denotes the set of integers composed only of $p, q$.

In this paper, we determine all prime powers $p^{e}$ with $N_{q}\left(p^{e}\right) \leq 4$ for small primes $p, q$. Indeed, we shall find all the solutions of the slightly generalized equation

$$
x^{e}=\epsilon_{1} y^{f_{1}}+\epsilon_{2} y^{f_{2}}+\epsilon_{3} y^{f_{3}}+\epsilon_{4} y^{f_{4}},
$$

in integers $e, \epsilon_{i} \in\{-1,0,1\}$ and $f_{1} \geq f_{2} \geq f_{3} \geq f_{4} \geq 0$, for relatively prime integers $2 \leq x, y \leq 100$. The case $(x, y)>1$ shall be discussed in the forthcoming papers.

Since the case $\epsilon_{1} \epsilon_{2} \epsilon_{3} \epsilon_{4}=0$ is dealt in [2], we deal only the case no subsum of the right of (1) vanishes (in particular, none of $\epsilon_{i}$ 's is equal to zero).

We begin by noting that we must have $f_{4}=0$ since $(x, y)=1$. Moreover, we find that $\epsilon_{1}$ must be positive. Assume that $\epsilon_{1}=-1$ and $f_{1}>f_{2}$. Then, we must have $x^{e} \leq$ $-y^{f_{1}}+y^{f_{1}-1}+y^{f_{1}-1}+1 \leq 1-y^{f_{1}-1}(y-2) \leq 1$, which is a contradiction. If $\epsilon_{1}=-1$ and $f_{1}=f_{2}$, then we must have $\epsilon_{2}=-1$.

Moreover, we may assume that $x, y$ are not perfect powers. Otherwise we can replace $e$ by $e l$ if $x=x_{0}^{l}$ for some $x_{0}, f \geq 2$ and we can replace $f_{i}$ 's by $f_{i} g$ if $y=y_{0}^{g}$ for some $y_{0}, g \geq 2$.

THEOREM 1.1. Let $2 \leq x, y \leq 100$ be relatively prime integers below 100 which are not perfect powers. Any solution of (1) satisfies one of the following conditions:

(1) The solution appears in Table 1. 
Table 1. Perfect powers representable as $y^{f_{1}} \pm y^{f_{2}} \pm y^{f_{3}} \pm y^{f_{4}}$

\begin{tabular}{|c|c|c|c|}
\hline$x^{e}$ & One of representations & $x^{e}$ & One of representations \\
\hline $2^{4}$ & $3^{2}+3^{2}-3^{0}-3^{0}$ & $15^{2}$ & $2^{7}+2^{7}-2^{5}+2^{0}$ \\
\hline $2^{4}$ & $3^{2}+3^{2}-3^{1}+3^{0}$ & $17^{2}$ & $2^{8}+2^{5}+2^{0}$ \\
\hline $2^{5}$ & $3^{3}+3^{1}+3^{1}-3^{0}$ & $17^{2}$ & $6^{3}+6^{2}+6^{2}+6^{0}$ \\
\hline $2^{5}$ & $3^{3}+3^{2}-3^{1}-3^{0}$ & $17^{2}$ & $12^{2}+12^{2}+12^{0}$ \\
\hline $2^{8}$ & $3^{5}+3^{2}+3^{1}+3^{0}$ & $18^{2}$ & $13^{2}+13^{2}-13^{1}-13^{0}$ \\
\hline $2^{9}$ & $3^{5}+3^{5}+3^{3}-3^{0}$ & $20^{2}$ & $7^{3}+7^{2}+7^{1}+7^{0}$ \\
\hline $2^{9}$ & $3^{6}-3^{5}+3^{3}-3^{0}$ & $20^{3}$ & $63^{2}+63^{2}+63^{1}-63^{0}$ \\
\hline $2^{5}$ & $5^{2}+5^{1}+5^{0}+5^{0}$ & $21^{2}$ & $2^{9}-2^{6}-2^{3}+2^{0}$ \\
\hline $2^{7}$ & $5^{3}+5^{1}-5^{0}-5^{0}$ & $22^{2}$ & $3^{5}+3^{5}-3^{1}+3^{0}$ \\
\hline $2^{8}$ & $5^{3}+5^{3}+5^{1}+5^{0}$ & $23^{2}$ & $2^{8}+2^{8}+2^{4}+2^{0}$ \\
\hline $2^{12}$ & $45^{2}+45^{2}+45^{1}+45^{0}$ & $23^{2}$ & $2^{9}+2^{3}+2^{3}+2^{0}$ \\
\hline $2^{13}$ & $91^{2}-91^{1}+91^{0}+91^{0}$ & $23^{2}$ & $2^{9}+2^{4}+2^{0}$ \\
\hline $3^{3}$ & $2^{5}-2^{1}-2^{1}-2^{0}$ & $23^{3}$ & $78^{2}+78^{2}-78^{0}$ \\
\hline $3^{3}$ & $2^{5}-2^{2}-2^{0}$ & $24^{2}$ & $17^{2}+17^{2}-17^{0}-17^{0}$ \\
\hline $3^{3}$ & $2^{4}+2^{3}+2^{2}-2^{0}$ & $26^{2}$ & $3^{6}-3^{4}+3^{3}+3^{0}$ \\
\hline $3^{3}$ & $2^{4}+2^{4}-2^{2}-2^{0}$ & $26^{3}$ & $3^{9}-3^{7}+3^{4}-3^{0}$ \\
\hline $3^{4}$ & $2^{6}+2^{4}+2^{0}$ & $26^{2}$ & $15^{2}+15^{2}+15^{2}+15^{0}$ \\
\hline $3^{5}$ & $2^{8}-2^{4}+2^{2}-2^{0}$ & $28^{2}$ & $3^{6}+3^{4}-3^{3}+3^{0}$ \\
\hline $3^{3}$ & $5^{2}+5^{0}+5^{0}$ & $28^{3}$ & $3^{9}+3^{7}+3^{4}+3^{0}$ \\
\hline $3^{5}$ & $11^{2}+11^{2}+11^{0}$ & $31^{2}$ & $2^{9}+2^{9}-2^{6}+2^{0}$ \\
\hline $5^{2}$ & $2^{3}+2^{3}+2^{3}+2^{0}$ & $31^{2}$ & $2^{10}-2^{6}+2^{0}$ \\
\hline $5^{2}$ & $2^{4}+2^{3}+2^{0}$ & $33^{2}$ & $2^{9}+2^{9}+2^{6}+2^{0}$ \\
\hline $5^{2}$ & $2^{5}-2^{3}+2^{0}$ & $33^{2}$ & $2^{10}+2^{6}+2^{0}$ \\
\hline $5^{3}$ & $2^{6}+2^{6}-2^{1}-2^{0}$ & $33^{2}$ & $10^{3}+10^{2}-10^{1}-10^{0}$ \\
\hline $5^{3}$ & $2^{6}+2^{6}-2^{2}+2^{0}$ & $39^{2}$ & $2^{10}+2^{9}-2^{4}+2^{0}$ \\
\hline $5^{3}$ & $2^{7}-2^{1}-2^{0}$ & $39^{2}$ & $2^{11}-2^{9}-2^{4}+2^{0}$ \\
\hline $5^{3}$ & $2^{7}-2^{2}+2^{0}$ & $41^{2}$ & $29^{2}+29^{2}-29^{0}$ \\
\hline $5^{4}$ & $2^{9}+2^{7}-2^{4}+2^{0}$ & $44^{2}$ & $3^{7}-3^{5}-3^{2}+3^{0}$ \\
\hline $5^{2}$ & $3^{3}+3^{0}+3^{0}$ & $45^{2}$ & $2^{11}-2^{4}-2^{3}+2^{0}$ \\
\hline $5^{2}$ & $3^{3}-3^{1}+3^{0}$ & $46^{2}$ & $3^{7}-3^{4}+3^{2}+3^{0}$ \\
\hline $7^{2}$ & $2^{4}+2^{4}+2^{4}+2^{0}$ & $47^{2}$ & $2^{11}+2^{7}+2^{5}+2^{0}$ \\
\hline $7^{2}$ & $2^{5}+2^{3}+2^{3}+2^{0}$ & $47^{2}$ & $13^{3}+13^{1}-13^{0}$ \\
\hline $7^{2}$ & $2^{5}+2^{4}+2^{0}$ & $49^{2}$ & $2^{6}-2^{4}+2^{0}$ \\
\hline $7^{2}$ & $2^{6}-2^{4}+2^{0}$ & $49^{2}$ & $2^{5}+2^{5}-2^{4}+2^{0}$ \\
\hline $7^{2}$ & $5^{2}+5^{2}-5^{0}$ & $53^{2}$ & $6^{4}+6^{4}+6^{3}+6^{0}$ \\
\hline $7^{3}$ & $18^{2}+18^{1}+18^{0}$ & $56^{2}$ & $5^{5}+5^{1}+5^{1}+5^{0}$ \\
\hline $7^{3}$ & $19^{2}-19^{1}+19^{0}$ & $56^{2}$ & $15^{3}-15^{2}-15^{1}+15^{0}$ \\
\hline $10^{2}$ & $3^{4}+3^{3}-3^{2}+3^{0}$ & $57^{2}$ & $5^{5}+5^{3}-5^{0}$ \\
\hline $10^{3}$ & $3^{6}+3^{5}+3^{3}+3^{0}$ & $58^{2}$ & $41^{2}+41^{2}+41^{0}+41^{0}$ \\
\hline $10^{2}$ & $7^{2}+7^{2}+7^{0}+7^{0}$ & $63^{2}$ & $2^{11}+2^{11}-2^{7}+2^{0}$ \\
\hline $11^{2}$ & $2^{6}+2^{6}-2^{3}+2^{0}$ & $63^{2}$ & $2^{12}-2^{7}+2^{0}$ \\
\hline $11^{2}$ & $2^{7}-2^{2}-2^{1}-2^{0}$ & $65^{2}$ & $2^{11}+2^{11}+2^{7}+2^{0}$ \\
\hline $11^{2}$ & $2^{7}-2^{2}-2^{2}+2^{0}$ & $65^{2}$ & $2^{12}+2^{7}+2^{0}$ \\
\hline $11^{2}$ & $2^{7}-2^{3}+2^{0}$ & $72^{2}$ & $17^{3}+17^{2}-17^{1}-17^{0}$ \\
\hline $11^{2}$ & $5^{3}-5^{1}+5^{0}$ & $80^{2}$ & $3^{8}-3^{5}+3^{4}+3^{0}$ \\
\hline $11^{3}$ & $6^{4}+6^{2}-6^{0}$ & $80^{3}$ & $3^{12}-3^{9}+3^{5}-3^{0}$ \\
\hline $11^{3}$ & $37^{2}-37^{1}-37^{0}$ & $82^{2}$ & $3^{8}+3^{5}-3^{4}+3^{0}$ \\
\hline $12^{2}$ & $5^{3}+5^{2}-5^{1}-5^{0}$ & $82^{3}$ & $3^{12}+3^{9}+3^{5}+3^{0}$ \\
\hline $13^{2}$ & $2^{7}+2^{5}+2^{3}+2^{0}$ & $89^{2}$ & $2^{13}-2^{8}-2^{4}+2^{0}$ \\
\hline $13^{3}$ & $3^{7}+3^{2}+3^{0}$ & $97^{2}$ & $56^{2}+56^{2}+56^{2}+56^{0}$ \\
\hline $15^{2}$ & $2^{8}-2^{5}+2^{0}$ & $99^{2}$ & $70^{2}+70^{2}+70^{0}$ \\
\hline
\end{tabular}


(2) There exists a pair of integers $w, g$ such that $e=2 g,(x, y)=\left(w, w^{g} \pm 1\right)$, $\left(f_{1}, f_{2}, f_{3}\right)=(2,1,1)$ and $\epsilon_{2}=\epsilon_{3}=\mp 1, \epsilon_{4}=1$.

(3) $e \leq 1$ or $f_{1} \leq 1$.

(4) The solution arises from the relations $2^{3}=3^{2}-3^{0}$ and $3^{2}=2^{3}+2^{0}$.

Our method is similar to the one of Deze, Tijdeman and Wang in its essence. Deze, Tijdeman and Wang used de Weger's lower bound for differences between two prime powers [9], which follows from lower bounds for linear forms in two logarithms and classical theory of continued fractions.

However, we need to deal linear forms of three logarithms of the form $e \log x$ $-f \log y-\log A$. In order to improve lower bounds for such linear forms, we use a variant of Davenport's method [1].

Our table suggests that an integer solution of (1) with $\min \left\{e, f_{1}\right\} \geq 3$ can be derived from one of the identities (a) $2^{3}=3^{f}-3^{f}+3^{2}-3^{0}$ and (b) $\left(3^{g} \pm 1\right)^{3}=$ $3^{3 g} \pm 3^{2 g+1}+3^{g+1} \pm 3^{0}$, except from finitely many ones.

2. Results on linear forms of logarithms. First, we introduce some notations. We denote by $\|\alpha\|$ the smallest distance between $\alpha$ and the integers and denote $v_{m}(n)$ by the largest integer $v$ such that $m^{v} \mid n$.

We use Matveev's lower bound for linear forms in logarithms, which is the best known result applicable for an arbitrary one.

THEOREM 2.1 (Matveev's theorem). Let $a_{1}, a_{2}, \ldots, a_{n}$ be non-zero integers such that $\log a_{1}, \ldots, \log a_{n}$ are not all zero. Let $A_{1}, \ldots, A_{n}$ be real numbers such that $A_{j} \geq$ $\max \left\{0.16, \log a_{j}\right\}$ for each $j$.

Put

$$
\Lambda=b_{1} \log a_{1}+\cdots+b_{n} \log a_{n}
$$

and

$$
\begin{aligned}
B & =\max \left\{1,\left|b_{1}\right| A_{1} / A_{n},\left|b_{2}\right| A_{2} / A_{n}, \ldots,\left|b_{n}\right|\right\}, \\
\Omega & =A_{1} A_{2}, \ldots, A_{n}, \\
C(n) & =\frac{16}{n !} e^{n}(2 n+3)(n+2)(4(n+1))^{n+1}\left(\frac{1}{2} e n\right)(4.4 n+5.5 \log n+7) .
\end{aligned}
$$

Then we have

$$
\log |\Lambda|>-C(n)(\log 3-\log 2+1+\log B) \max \left\{1, \frac{n}{6}\right\} \Omega .
$$

Although Matveev's theorem gives an effective bound for solutions of our equation, this bound is fairly large. We wish to obtain a stronger lower bounds to $|\Lambda|$ for coefficients below an upper bound given by Matveev's theorem. Davenport's lemma is useful for this purpose (see [1]). We use the following formulation.

THEOREM 2.2 (Davenport's lemma). Let $\beta, \theta_{1}, \theta_{2}$ be real numbers such that $\theta=-\theta_{1} / \theta_{2}$ is irrational. Let $x_{1}, x_{2}, X_{0}$ be integers and $X=\max \left\{x_{1}, x_{2}\right\}$. Put $\Lambda$ $=\beta+x_{1} \theta_{1}+x_{2} \theta_{2}$ and $\psi=\beta / \theta_{2}$.

If a convergent $p / q$ of $\theta$ with $q>X_{0}$ satisfies the inequality $\|q \psi\|>2 X_{0} / q$, then $|\Lambda|>X_{0}\left|\theta_{2}\right| / q^{2}$. 

obtain

Proof. Using the fact that $q\left(x_{1} \theta-x_{2}\right)-x_{1}(q \theta-p)=p x_{1}-q x_{2}$ is an integer, we

$$
\|q \psi\|=\left\|q \Lambda / \theta_{2}+q\left(x_{1} \theta-x_{2}\right)\right\|=\left\|q \Lambda / \theta_{2}+x_{1}(q \theta-p)\right\| .
$$

We can easily see that this is at most

$$
q\left|\Lambda / \theta_{2}\right|+\left|x_{1}\right| / q \leq q\left|\Lambda / \theta_{2}\right|+X_{0} / q
$$

From Theorems 94 and 95 in Nagell [4], we obtain the following lemma.

LEMMA 2.3. For any odd prime q dividing $y$, we have

$$
v_{y}\left(x^{e}-1\right) \leq\left(v_{q}(e)+v_{q}\left(x^{q-1}-1\right)\right) / v_{q}(y)
$$

and

$$
v_{y}\left(x^{e}+1\right) \leq\left(v_{q}(e)+v_{q}\left(x^{q-1}-1\right)\right) / v_{q}(y)
$$

Moreover, we have

$$
v_{y}\left(x^{e}-1\right) \leq\left(v_{2}(e)+v_{2}\left(x^{2}-1\right)-1\right) / v_{2}(y)
$$

and

$$
v_{y}\left(x^{e}+1\right) \leq\left(v_{2}\left(x^{2}-1\right)\right) / v_{2}(y) .
$$

Let $a(x, y)=\min v_{q}\left(x^{2(q-1)}-1\right) / v_{q}(y)$ over primes $q$ dividing $y$. We can confirm by computation that $v_{q}\left(x^{2(q-1)}-1\right) \leq 6$ for any prime $q<100$ and integer $x \leq 100$. This immediately gives the following result.

COROLlary 2.4. If $x, y<100$, then we have

$$
v_{y}\left(x^{e} \pm 1\right) \leq a(x, y)+\log e / \log y
$$

and

$$
v_{y}\left(x^{e} \pm 1\right) \leq 6+\log e / \log y .
$$

3. Proof of main theorem. Throughout this section, we let $x, y$ be relatively prime integers less than 100 which are not perfect powers and put $A=y^{f_{1}-f_{2}}+\epsilon_{2}, \Lambda_{1}=$ $e \log x-f_{1} \log y, \Lambda_{2}=e \log x-f_{2} \log y-\log A$.

We shall give the first upper bound using Matveev's theorem.

LEMMA 3.1. If $\left(e, f_{1}, f_{2}, f_{3}\right)$ is a solution of $(1)$, then $e<3 \times 10^{21}$ and $f_{1}<3 \times$ $10^{21} \log x / \log y$.

Proof. Assume that $\left(e, f_{1}, f_{2}, f_{3}, \epsilon_{2}, \epsilon_{3}, \epsilon_{4}\right)\left(\epsilon_{i} \in\{-1,0,1\}, f_{i} \geq 0\right)$ is a solution of (1) such that the right-hand side of (1) has no vanishing subsum.

First we shall obtain an upper bound for $A$. By (1), we have $y^{f_{2}} \geq\left|x^{e}-y^{f_{1}}\right| / 2-1$. Since $\left|x^{e}-y^{f_{1}}\right|>y^{f_{1}}\left|\Lambda_{1}\right| / 2$, we have $y^{f_{2}}>y^{f_{1}}\left|\Lambda_{1}\right| / 5$. This gives

$$
|A| \leq y^{f_{1}-f_{2}}+1 \leq 1+5 /\left|\Lambda_{1}\right| \leq \exp (2) /\left|\Lambda_{1}\right| .
$$


Now we apply Theorem 2.1 to estimate $\Lambda_{1}$. We have

$$
\log |A| \leq 2+C(2)\left(\log 3-\log 2+1+\log B_{1}\right) \log x \log y,
$$

where $B_{1}=\max \left\{e \log x / \log y, f_{1}\right\}$ if $x<y$ and $B_{1}=\max \left\{e, f_{1} \log y / \log x\right\}$ if $x>y$.

Since the right-hand side of (1) has no vanishing subsum, we have $y^{f_{1}} \leq 8 x^{e}$. Therefore we obtain

$$
\log |A| \leq 2+C(2)(\log 3-\log 2+1+\log (e+3)) \log x \log y .
$$

Put $A^{\prime}=\exp (2+C(2)(\log 3-\log 2+1+\log (e+3)) \log x \log y)$, so that the inequality $|A| \leq A^{\prime}$ holds.

Second we shall obtain a lower bound for $f_{3}$. We can easily see that

$$
y^{f_{3}} \geq x^{e}-y^{f_{1}}-y^{f_{2}}-1 \geq x^{e}-A y^{f_{2}}-1 \geq x^{e}\left|\Lambda_{2}\right| / 3 .
$$

Applying Theorem 2.1 again, we have

$$
\log \left|\Lambda_{2}\right| \geq W \log x \log y \log A^{\prime},
$$

where

$$
\begin{gathered}
W=C(3)\left(\log 3-\log 2+1+\log B_{2}\right), \\
B_{2}=\max \left\{e \log x / \log A^{\prime}, f_{2} \log y / \log A^{\prime}, 1\right\} .
\end{gathered}
$$

Combining (16) and (17), we have

$$
f_{3} \geq \frac{e \log x-\log 3}{\log y}-C(3)\left(\log 3-\log 2+1+\log B_{2}\right) \log x \log A^{\prime} .
$$

On the other hand, Corollary 2.4 gives

$$
f_{3}=v_{y}\left(x^{e} \pm 1\right) \leq 6+\log e / \log y .
$$

It follows from (16), (17) and (21) that

$$
6 \log y+\log 3+\log e+W(\log x)(\log y)\left(\log A^{\prime}\right)-e \log x \geq 0 .
$$

For each pair of relatively prime integers $x, y$ below 100 , we see that the left-hand side of (22) is negative for $e=2.9 \times 10^{21}$ and decreasing for $e \geq 2.9 \times 10^{21}$. This proves the lemma.

LEMMA 3.2. If $\left(e, f_{1}, f_{2}, f_{3}\right)$ is a solution of (1), then we have $\left|\Lambda_{1}\right| \geq 10^{-27}$ and $y^{f_{1}-f_{2}}<5 \times 10^{27}$.

Proof. Let $p_{n} / q_{n}$ be the $n$th convergent of $\log x / \log y$. We set $n$ to be the smallest index for which $q_{n}>3 \times 10^{21}$. We confirmed by computation that $\left|q_{n} \log x-p_{n} \log y\right| \geq 10^{-27}$ for all relatively prime integers $x, y$ below 100 . By Lemma 3.1, we have $e<3 \times 10^{21} \leq q_{n}$. Hence, by Theorem 184 of [3], we have $\left|\Lambda_{1}\right|=\left|e \log x-f_{1} \log y\right| \geq\left|q_{n} \log x-p_{n} \log y\right|$. Since we have $y^{f_{2}}>y^{f_{1}}\left|\Lambda_{1}\right| / 5$, we have $y^{f_{1}-f_{2}}<5 \times 10^{27}$.

LEMMA 3.3. If $\left(e, f_{1}, f_{2}, f_{3}\right)$ is a solution of $(1)$, then we have $x^{e} \geq 3 \times 10^{35} y^{f_{3}}$. 
Proof. We distinguish two cases according to whether $A=x^{g}$ for some integer $g \geq 0$ or not.

Assume that $A=x^{g}$ for some integer $g \geq 0$. Then we have $x^{e}=A y^{f_{2}} \pm y^{f_{3}} \pm 1$. Hence $y^{f_{3}} \geq\left|x^{e}-A y^{f_{2}}\right|-1 \geq x^{g}\left|x^{e-g}-y^{f_{2}}\right|-1$. Similarly, with Lemma 3.2, we obtain that $\left|(e-g) \log x-f_{2} \log y\right|>10^{-27}$ and therefore $y^{f_{3}} \geq 10^{-28} x^{e}$.

Assume that $A$ is not of the form $x^{g}$ with $g$ a non-negative integer. By definition, $A$ is an integer of the form $y^{f} \pm 1$, where $f$ is a non-negative integer satisfying $y^{f}<5 \times 10^{27}$, but not of the form $x^{g}$ with $g$ a non-negative integer. For all of such integers, we apply Davenport's method to obtain a lower bound for $\left|\Lambda_{2}\right|$ with $e<3 \times 10^{21}$. Put $\beta=\log A, \theta_{1}=\log y, \theta_{2}=\log x, X_{0}=3 \times 10^{21} \log x / \log y$ and apply Theorem 2.2 for all $x, y, A=y^{f} \pm 1$ with $(x, y)=1,2 \leq x, y<100$ and $f<\log \left(5 \times 10^{27}\right) / \log y$.

Computation shows that we can apply Theorem 2.2 with an appropriate convergent $p / q$ of $\theta=-\theta_{1} / \theta_{2}$ and we have the inequality $\left|\Lambda_{2}\right| \geq 10^{-35}$ for all $x, y, A$ satisfying this condition. It follows from (16) that $y^{f_{3}} \geq x^{e}\left|\Lambda_{2}\right| / 3 \geq 10^{-35} x^{e} / 3$.

Now we prove Theorem 1.1. By Lemma 3.3, we have

$$
x^{e} \leq 3 \times 10^{35} e y^{a(x, y)}
$$

by Corollary 2.4 .

Computation shows that $a(x, y) \leq 6$ for $x<100, y=2, a(x, y) \leq 4$ for $x<$ $100, y=3, a(x, y) \leq 3$ for $x<100,5 \leq y \leq 23$ and $a(x, y) \leq 2$ for $x<100,24 \leq y<$ 100. Hence (23) gives that $e \leq 138$.

Computer search over all integers of the form $x^{e}$ with $x<100, e \leq 138$ revealed that for any relatively prime integers $x, y<100$, all solutions of (1) with $e, f_{1} \geq 2$ are derived from the identities given in the statement of the theorem except those given in Table 1. This completes the proof.

\section{REFERENCES}

1. A. Baker and H. Davenport, The equations $3 x^{2}-2=y^{2}$ and $8 x^{2}-y=z^{2}$, Quart. $J$. Math. 20 (1969), 129-137.

2. M. Dede and R. Tijdeman, Exponential diophantine equations with four terms, Indag. Math. N.S. 3 (1992), 47-57.

3. G. H. Hardy and E. M. Wright, An introduction to the theory of numbers, 5 th ed. (The Claredon Press, Oxford University Press, New York, 1979). 1964).

4. T. Nagell, Introduction to number theory, 2nd ed. (Chelsea Publishing Co., New York,

5. H. G. Senge and E. G. Strauss, P. V. numbers and sets of multiplicity, Period Math. Hungar. 3 (1973), 93-100.

6. C. L. Stewart, On the representation of an integer in two different bases, J. Reine. Angew. Math. 319 (1980), 63-72.

7. R. Tijdeman and Wang Lianxiang, Sums of products of powers of given prime numbers, Pacific J. Math. 132 (1988), 177-193, Corr. 135 (1988), 396-398.

8. Wang Lianxiang, Four terms equations, Indag. Math. $\mathbf{5 1}=$ Proc. K. N. A. W. Ser. A 92 (1989), 355-361.

9. B. M. M. de Weger, Solving exponential diophantine equations using lattice basis reduction algorithms, J. Number Theory 26 (1987), 325-367. 